Proceedings of the 1997 IEEE

International Conference on Robotics and Automation

Albuquerque, New Mexico - April 1997

\title{
Sensor Based Planning for a Planar Rod Robot: Incremental Construction of the Planar Rod-HGVG
}

\author{
Howie Choset and Brian Mirtich and Joel Burdick
}

\begin{abstract}
This work considers sensor based motion planning for rod-shaped robots in unknown environments. The motion planning scheme is based on the rod hierarchical generalized Voronoi graph (rod-HGVG). The rod-HGVG is a roadmap for rod-like robots, and is an extension of a prior roadmap for point-like robots. We give an incremental method to construct the rodHGVG, thereby enabling exploration of unknown environments. An important practical feature of the algorithm is its sole reliance upon the use of work space distance measurements to objects that are within line of sight. Such measurements can be readily provided by conventional range sensors. Moreover, motion planning in a configuration space is achieved without explicitly constructing each configuration space obstacle. A key result derived in this paper is the distance gradient between two convex sets.
\end{abstract}

\section{Introduction}

Sensor based planning makes use of sensor information reflecting the current state of the environment, in contrast to classical planning, which assumes full knowledge of the environment prior to planning. Recent work [4] includes the definition of a new roadmap structure termed the rod hierarchical generalized Voronoi graph (rod-HGVG) which serves as a basis for sensor based planning for a rod shaped robot operating in the plane.

Recall that a roadmap is a geometric structure that captures the global topological properties of the robot's free space and has the following important properties: accessibility, connectivity, and departability. These properties imply that the planner can construct a path between any two points in a connected component of the robot's free space by first finding a path onto the roadmap (accessibility), traversing the roadmap to the vicinity of the goal (connectivity), and then constructing a path from the roadmap to the goal (departability).

The focus of current work is on the incremental construction of the rod-HGVG using only line of sight range data. An incremental construction procedure is necessary for sensor based planning because most environments do not have one vantage point from which the robot can "see" the entire environment. Therefore, the robot must coordinate motion and sensing to explore an unknown environment.

The primary advantage of rod-HGVG incremental construction procedure is that distance measurements are made entirely in the workspace, instead of the con- figuration space where measuring distance with conventional sensors is quite difficult. In fact, the incremental construction procedure for the rod robot bypasses the need for the explicit construction of the configuration space. While the primary intention is to use the rodHGVG as a basis for sensor based planning, it can also be used for classical motion planning.

The rod-HGVG definitions are based upon the definitions of the HGVG, a roadmap structure that serves as the basis for sensor based planning for a point robot [2]. Robots that can be modeled as a point include most cicular symetric mobile robot bases. Rod robots are useful for motion planning of long and narrow delivery robot systems and robot blimps; however this work is the next step towards the ultimate goal of sensor based planning for an articulated multi-body chain robot. To better distinguish the two roadmaps, term the point based HGVG as the point-HGVG.

\section{Relation to Prior Work}

Sensor based planning has received increased attention, as it is a requirement for realistic deployment of autonomous robots in unstructured environments. For a review of many sensor based planning techniques, see [14]. Unfortunately, current sensor based planning methods are limited because: (1) many are based on heuristic algorithms, and it is therefore impossible to prove if they will work in all possible environments; (2) proof of convergence for other algorithms is limited to the case of a point in two-dimensional environments (for example, Lumelsky's "bug" algorithm [8]); or (3) the robot is assumed to be a point in configuration space, where its sensors can measure distance in configuration space.

The rod-HGVG is based upon the generalized Voronoi diagram (GVD) which was aplied to motion planning of a disk by Ó'Dúnlaing and Yap [12]. However, the method in [12] requires full knowledge of the world's geometry prior to the planning event. This work was extended to the case where the robot is a rod in [11], but it, too, requires full knowledge of the world's geometry prior to the planning event. Recently, Cox and Yap [6] developed an "on-line" strategy for path planning for rods. Although this method can be readily modified with tactile sensors for sensor based use, it does not provide a roadmap of the rod robot's free space.

To our knowledge, the only sensor based adaptations of roadmaps for configuration space dimensions greater 
than two are [15] (which is based on Canny and Lin's Opportunistic Path Planner (OPP) [1]), and [2] (which is where the hierarchical generalized Voronoi graph (HGVG) is defined). A limitation of these roadmaps is that distance measurements are assumed to be made in a configuration space (or some parameterization of it). Previous work [4] introduces the rod-HGVG, which is a configuration space roadmap defined in terms of workspace distance measurements which are obtainable from realistic sensors.

This paper focuses on the incremental construction procedure of the rod-HGVG. The approach, described in this paper, is based on the point-HGVG incremental construction procedure [3]. The advantage of the point-HGVG incremental procedure is that it uses only line of sight information and functions in non-planar environments. The drawback of the point-HGVG procedure is that it assumes distance measuresments are made in a configuration space (or some parameterization of it). The rod-HGVG incremental construction procedure requires only work space distance measurements, and thus, the rod-HGVG is easy to construct using realistic sensors.

\begin{tabular}{ccccc|}
\hline point-GVD & $\rightarrow$ & point-GVG & $\rightarrow$ & point-HGVG \\
& & & & $\downarrow$ \\
rod-GVD & $\rightarrow$ & rod-GVG & $\rightarrow$ & rod-HGVG
\end{tabular}

\section{The Generalized Voronoi Graph}

Since this work is heavily based upon the point based GVG, this section is dedicated to the generalized Voronoi graph (GVG) [2]. The GVG is the foundation for a point robot roadmap (i.e., the point-HGVG). In this paper, we term the GVG the point-GVG in order to distinguish it from the generalized Voronoi graph for a rod, which is defined in a later section.

The point-GVG is defined in terms of a distance function

$$
d_{i}(r)=\min _{c \in C_{i}}\|r-c\|
$$

where $r \in \mathbb{R}^{m}$ and $C_{i}$ is a convex obstacle. The basic building block of the point-GVG is the two-equidistant surjective surface which is the set of points equidistant to two convex obstacles, and is denoted

$S \mathcal{S}_{i j}=\left\{r \in \mathbb{R}^{m}:\left(d_{i}-d_{j}\right)(r)=0\right.$ and $\left.\nabla d_{i}(r) \neq \nabla d_{j}(r)\right\}$, where $\nabla d_{i}(r)$ is a unit vector based at $r$ and pointing away from the minimizing $c$ along a line defined by $c$ and $r$ (see Equation 1). In constructing the point-GVG, we are interested in a subset of $S S_{i j}$ termed the twoequidistant face which is defined as

$$
\mathcal{F}_{i j}=\left\{r \in \operatorname{cl}\left(\mathcal{S} \mathcal{S}_{i j}\right): d_{i}(r) \leq d_{h}(r) \forall h\right\} .
$$

The intersection of $\mathcal{F}_{i j}$ and $\mathcal{F}_{j k}$ forms a three-equidistant face, denoted $\mathscr{F}_{i j k}$, and it is the set of points equidistant and closest to three obstacles: $C_{i}, C_{j}$, and $C_{k}$ [2].

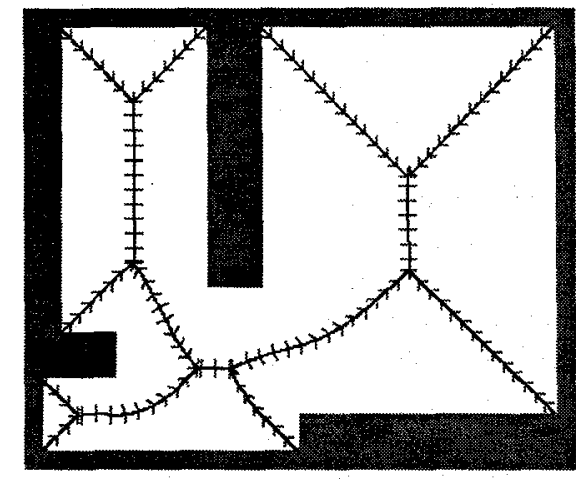

Fig. 1. The ticked line segments form the planar point-GVG for a bounded environment. The ticks point at the nearest point on an obstacle, and are thus the negated gradients of the distance function.

In the plane, the two-equidistant faces and threeequidistant faces are one and zero-dimensional, respectively and the point-GVG is the collection of these onedimensional edges and zero-dimensional vertices. In the plane, the point-GVG, which is the set of points equidistant to two or more closest obstacles, is always connected. See Figure 1. (In $\mathbb{R}^{m}$, the point-GVG is the set of points equidistant to $m$ obstacles and is always one-dimensional, though not necessarily connected [2].)

A key feature of the point-GVG is that it can be incrementally constructed using line of sight range data. This incremental construction procedure is described in [3].

\section{Rod Distance Function}

Definition 4.1 (RoD) A rod $R$ is a line segment of length $L$ that has two end points $P$ and $Q$.

The configuration space of the rod is $S E(2)(S E(2) \simeq$ $\mathbb{R}^{2} \times S^{1}$ ). Let $q$ be the configuration of the rod, and let it be determined by the $x$ and $y$ coordinates of the point $P$, and the orientation of the rod with respect to the horizontal, i.e. $q=(x, y, \theta)$. See Figure 2. Let $P(q)$ be the $x$ and $y$ coordinates of the point $P$ when the rod is at configuration $q$, let $\theta(q)$ be the orientation of the rod when it is at configuration $q$, and let $R(q)$ be the set of points in the plane that the rod occupies when it is at configuration $q$. Note that $P(q) \in \mathbb{R}^{2}, \theta(q) \in S^{1}$, and $R(q) \subset \mathbb{R}^{2}$. Let superscripts ${ }^{x}$ and ${ }^{y}$ denote the $x$ and $y$ coordinates, respectively, of a point in the plane. For example, $P(q)^{x}$ is the $x$ coordinate of the point $P$ at configuration $q$. Finally, when $X \subset \mathbb{R}^{2}$, let $q(X)$ be the set of configurations $q^{*}$ where $R\left(q^{*}\right) \subset X$.

Assume a rod robot $R$ is operating in a subset $\mathcal{W}$ of $\mathbb{R}^{2} . \mathcal{W}$ is populated by obstacles $C_{1}, \ldots, C_{n}$ which are 


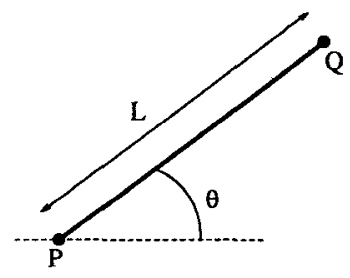

Fig. 2. The configuration of a rod is determined by the $x$ and $y$ coordinates of $P$ and the orientation of the rod with respect to the horizontal.

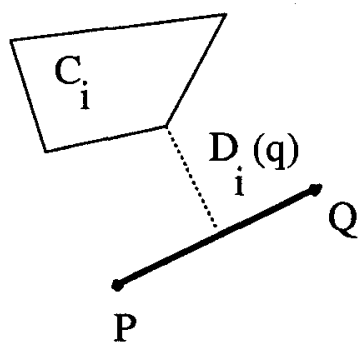

Fig. 3. The distance from the rod (thick solid line) to an obstacle is the distance (dotted line) between the nearest point on the rod to the obstacle and the nearest point on the obstacle to the rod.

convex sets. Non-convex obstacles are modeled as the union of convex shapes. It is assumed that the boundary of $\mathcal{W}$ is a collection of convex sets, which are members of the obstacle set $\left\{C_{i}\right\}$.

\section{Definition 4.2 (Rod Single ObJect Distance)}

The rod single object distance function is the distance between an obstacle $C_{i}$ and $a \operatorname{rod} R$ when the rod is at a configuration $q$. It is determined by

$$
D_{i}(q)=\min _{r \in R(q), c \in C_{i}}\|r-c\| \text {. }
$$

An important characteristic of $D_{i}(q)$ is that it can be readily computed from sensor measurements made in the workspace. For example, the rod robot in Figure 3 may have range sensors distributed around its perimeter. The distance between the obstacle and the rod is the measurement of the range sensor associated with a local minima of measurements. It can be shown that the rod-distance function is continuous and smooth in the interior of the workspace for convex sets.

The discussion of the gradient $\nabla D_{i}(q)$ is defined in a later section. However, it should be noted that $\nabla D_{i}(q)$ is continuous for convex sets.

\section{Rod-HGVG}

The rod-GVG, defined below, serves as a basis for the rod-HGVG. A key feature of the rod-GVG is that it is defined in terms of a distance function. The basic building block of the rod-GVG is the configuration two-equidistant face which is the set of rod configurations equidistant to two obstacles such that these two obstacles are the closest obstacles and no two gradient

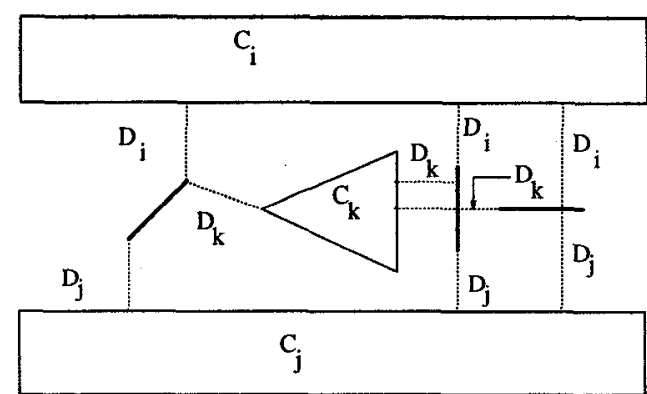

Fig. 4. The thick solid lines represent three configurations of the rod whose configurations are in the configuration threeequidistant face defined by obstacles $C_{i}, C_{j}$ and $C_{k}$. The thin dotted lines represent the distance between the rods and the obstacles.

vectors are co-linear. That is,

$$
\begin{aligned}
\mathcal{E F}_{i j}=\operatorname{cl}\{q \in S E(2): 0 & \leq D_{i}(q)=D_{j}(q) \leq D_{h}(q) \forall h \\
& \text { and } \left.\nabla D_{i}(q) \neq \nabla D_{j}(q)\right\}
\end{aligned}
$$

In the planar case, the rod-GVG edges are configuration three-equidistant faces and the the rod-GVG nodes are configuration four-equidistant faces. A configuration three-equidistant face, denoted $\mathrm{CF}_{i j k}$, is the set of rod configurations that are equidistant to three obstacles such that (1) these three obstacles are the closest obstacles and (2) no two gradient vectors to any of the three obstacles are co-linear. That is,

$$
\mathcal{E F}_{i j k}=\mathcal{E F}_{i j} \bigcap \mathcal{E F}_{i k} \bigcap \mathcal{E F}_{j k}
$$

Note that we only consider configuration threeequidistant faces that comprise the transversal intersection of configuration two-equidistant faces (a stability and generic assumption described in [2], [5]). The configuration four-equidistant faces are defined in a similar fashion. In the planar case, the configuration threeequidistant faces are the rod-GVG edges and the configuration four-equidistant faces are the rod-GVG vertices.

DEFINITION 5.1 (RoD-GVG) The collection of rodGVG edges and of rod-GVG vertices is the rod-GVG.

Alas, the rod-GVG is not necessarily connected as can be seen in Figure 5. In order to connect the rod-GVG, we define additional structures, termed $R$-edges, which link disconnected rod-GVG edges by exploiting the property that the point GVG is connected in the plane. See Figure 6 . The $R$-edges (Figure 7 ) are the set of rod configurations, $q$, whose $R(q)$ are in the tangent space of a point-GVG edge at a point, $r$, such that (I) $r \in R(q)$ and $r$ is closer to obstacles $C_{i}$ and $C_{j}$ than any other point on the rod and (II) no other obstacle is closer to the rod than the two equidistant obstacles. In other 


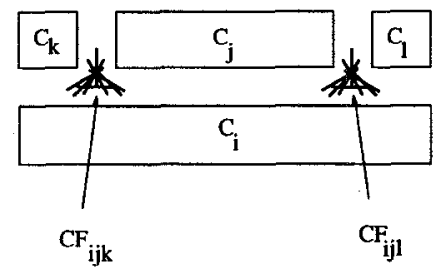

Fig. 5. The two clusters of solid lines represent rods whose configurations are triply equidistant to three obstacles. The left cluster represents rods whose configurations are elements of the rod-GVG edge $\mathrm{eF}_{i j k}$, and the right cluster are elements of $\mathrm{eF}_{i k l}$. In this example, both rod-GVG edges are diffeomorphic to $S^{1}$ (i.e., they are cyclic) and neither rod-GVG edge is connected to any other rod-GVG edge.

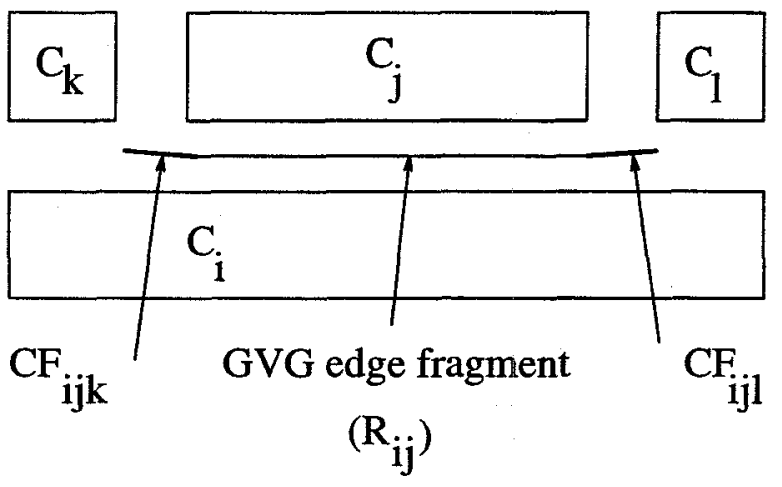

Fig. 6. The dark line segment on the left represents a rod configuration in $\mathrm{CF}_{i j k}$, and the dark segment on the right represents a rod configuration in $\mathrm{eF}_{i j l}$. These rods are connected by the point GVG edge $\mathcal{F}_{i j}$. The point GVG edge gives rise to a linking structure termed the $R$-edge which connects $\mathcal{E F}_{i j k}$ and $\mathrm{eF}_{i j l}$.

words.

$$
\begin{aligned}
\mathcal{R}_{i j}= & \left\{q \in \operatorname{cl}\left(q\left(T_{r} \mathcal{F}_{i j}\right)\right): r \in \mathcal{F}_{i j}\right. \text { and } \\
& (\mathrm{I}) 0 \leq d_{i}(r) \leq d_{i}\left(r_{1}\right) \forall r_{1} \in R(q) \text { and } \\
& \left.(\mathrm{II}) d_{i}(r) \leq D_{h}(q) \forall h \neq i, j\right\}
\end{aligned}
$$

In $S E(2)$, an $R$-edge is one-dimensional [4].

DEFINITION 5.2 (RoD-HGVG) The rod hierarchical generalized Voronoi graph (rod-HGVG) is the collection of rod-GVG edges and $R$-edges.

In the planar case, the rod-HGVG is accessible, connected, and departable [4]. Current work includes extending the rod-HGVG to the three-dimensional case.

\section{Incremental Construction}

\subsection{Incremental Accessibility}

It was shown in [4] that accessibility is achieved in two steps. Assume the robot starts at a point closest to a single object $C_{i}$. Boundedness of the workspace guarantees that a rod robot that follows a path traced by $\nabla D_{i}(q)$ arrives at a configuration that is equidistant to two objects, $C_{i}$ and $C_{j}$. Then, maintaining double equidistance, the robot increases distance from objects $C_{i}$ and $C_{j}$. Again, boundedness guarantees that the

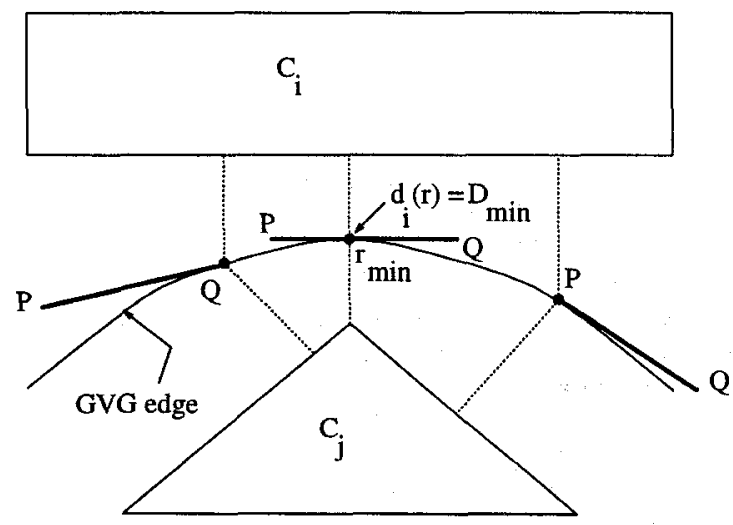

Fig. 7. The rod is moving from left to right while remaining tangent to the point GVG edge defined by obstacles $C_{i}$ and $C_{j}$. The thick solid lines represent different configurations of the rod in an $R$-edge. The dotted lines represent the shortest distance between the rod and the nearby obstacles. Note, for all configurations where the rod is tangent to $\mathcal{F}_{i j}$ but not at $r_{\min }$, the closest point on the rod to $C_{i}$ and $C_{j}$ is either $P$ or $Q$.

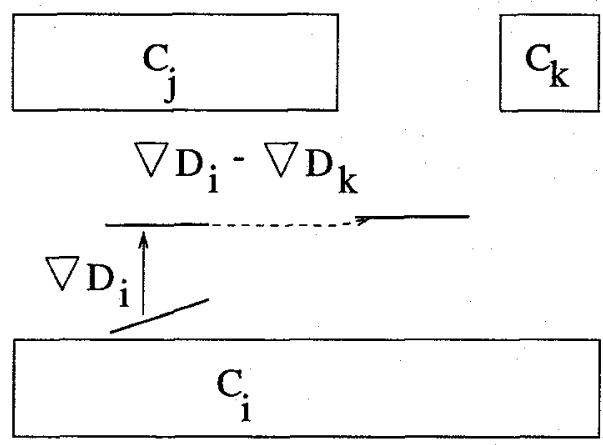

Fig. 8. The solid lines represent the rod, which is moving from an initial configuration to the rod-GVG. The solid arrow represents the portion of the path in which the rod is following the gradient to the nearest obstacle. The dotted arrow corresponds to the final portion of the path where the rod moves toward $C_{k}$ while remaining equidistant to $C_{i}$ and $C_{j}$ (i.e., the rod follows $\left.\pi_{T_{q}} \mathrm{eF} F_{i j} \nabla D_{i}(q)-\pi_{T_{q}} \mathrm{eF} \mathcal{F}_{i j} \nabla D_{k}(q)\right)$.

robot arrives at a configuration that is equidistant to three obstacle, i.e., a configuration on a rod-GVG edge. A key feature of this procedure is that it requires only line of sight information. See Figure 8.

\subsection{Traceability}

In an incremental context, the property of connectivity is interpreted as traceability. More specifically, traceability implies that using only local data, the robot can: (1) "trace" the point-GVG edges; (2) determine when to terminate the edge tracing process, and (3) determine when to start new edge tracing procedures.

\subsubsection{Rod-GVG edges}

As described in Section 5, there are two structures that compose the planar rod-HGVG: rod-GVG edges and $R$-edges. Both of these structures can be incremen- 
tally constructed using only line of sight information in a fashion similar to the incremental construction of pointGVG edges described in [3]. Just like the point-GVG edges, the rod-GVG edges are traced in an incremental manner using an adaptation of numerical continuation techniques [7].

Let $z_{1}$ be the basis of the tangent space of a configuration $q$ in the rod-GVG edge and let $z_{1}, z_{2}, z_{3}$ be the tangent space of $q$ in $S E(2)$. That is, $z_{1}, z_{2}, z_{3}$ can be viewed as a coordinate frame whose origin is located at $q$. Let $\lambda$ be a parameter which represents a displacement in the $z_{1}$ direction and let $y$ be the plane spanned by $z_{2}$ and $z_{3}$. This plane is termed the "normal plane" and is orthogonal to $z_{1}$, the tangent of the rod-GVG. Incremental construction of the rod-GVG edge is achieved by tracing the roots of the expression $G_{\operatorname{rod}}(y ; \lambda)=0$ as the parameter $\lambda$ varies.

$$
G_{\operatorname{rod}}(y ; \lambda)=\left[\begin{array}{l}
D_{i}(y ; \lambda)-D_{j}(y ; \lambda) \\
D_{i}(y ; \lambda)-D_{k}(y ; \lambda)
\end{array}\right]
$$

The function $G_{\text {rod }}(y ; \lambda)$ assumes a zero value only on a point-GVG edge. Hence, if the Jacobian of $G_{\text {rod }}$, which is

$$
\nabla_{y} G_{\mathrm{rod}}(y ; \lambda)=\left[\begin{array}{c}
\left(\nabla D_{i}(y ; \lambda)-\nabla D_{j}(y ; \lambda)\right)^{T} \\
\left(\nabla D_{i}(y ; \lambda)-\nabla D_{k}(y ; \lambda)\right)^{T}
\end{array}\right]
$$

is surjective, then the implicit function theorem asserts that the roots of $G_{\text {rod }}(y ; \lambda)$ locally define a rod-GVG edge as $\lambda$ is varied. A rod-GVG edge is constructed by numerically tracing the roots of $G$.

(In actuality, we are using the differential, not the gradient, in Equation 7, but for the sake of discussion, when we state gradient, we mean differential. Note that there is a natural relationship between the two. Discussion of the gradient appears in Section 7)

The explicit edge construction procedure has two steps: a predictor step and a corrector step. The predictor step moves the robot for a small distance along the tangent of the rod-GVG. The tangent direction is the null space of $\nabla_{y} G_{\text {rod }}[16]$. Since $\nabla_{y} G_{\text {rod }}$ comprises distance information, it can be readily computed with line of sight sensor information.

Typically, the prediction step takes the robot off of a rod-GVG edge, so a correction procedure is required to bring the robot back to the rod-GVG. If step size along the tangent is "small," then the graph will intersect a "correcting plane" (Figure 9), which is a plane orthogonal to the tangent. The correction step finds the location where the rod-GVG intersects the correcting plane (Figure 9) and is achieved via a iterative Newton's Method. If $y^{k}$ and $\lambda^{k}$ are the $k$ th estimates of $y$ and $\lambda$, the $k+1$ st iteration is defined as

$$
y^{k+1}=y^{k}-\left(\nabla_{y} G_{\mathrm{rod}}\right)^{-1} G_{\mathrm{rod}}\left(y^{k}, \lambda^{k}\right)
$$

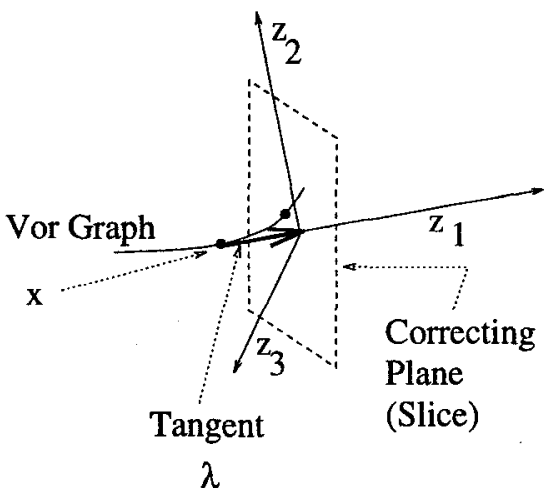

Fig. 9. Sketch of continuation method.

where $\nabla_{y} G_{\text {rod }}$ is the Jacobian of $G_{\text {rod }}$ restricted to the correcting plane evaluated at $\left(y^{k}, \lambda^{k}\right)$.

Now, it needs to be shown that:

Proposition 6.1 The matrix $\nabla_{y} G_{\text {rod }}$ is invertible.

Proof: Assume that $\nabla_{y} G_{\text {rod }}$ is not invertible. That is, assume $T_{q} \mathrm{eF}_{i j}=\kappa T_{q} \mathrm{eF}_{i k}$. By definition, for all $w \in T_{q} \mathrm{eF}_{i j},\left\langle\nabla\left(D_{i}(q)-D_{j}(q)\right), w\right\rangle=0$. Since $T_{q} \mathrm{eF}_{i j}=$ $\kappa T_{q} \mathrm{eF}_{i k}$, for all $w \in T_{q} \mathrm{eF}_{i j},\left\langle\nabla\left(D_{i}(q)-D_{k}(q)\right), w\right\rangle=0$. This implies that $T_{q} \mathrm{eF}_{i j}=T_{q} \mathrm{eF}_{i k}$ which violates the assumption that $\mathrm{eF}_{i j}$ and $\mathrm{eF}_{i k}$ transversally intersect. Therefore, $T_{q} \mathrm{eF}_{i j} \neq \kappa T_{q} \mathrm{eF}_{i k}$, and $\nabla_{y} G_{\text {rod }}$ is invertible.

Since $\nabla_{y} G_{\text {rod }}$ is invertible, Equation (8) is well posed. Practically speaking, this result states that the numerical procedure defined by Equation (8) will be robust for reasonable errors in robot position, sensor errors, and numerical round off.

\subsection{2 $R$-edges}

The incremental construction of the $R$-edges requires a slight modification of the already existing point-GVG incremental edge construction procedure [3]. The prediction step is identical: the rod robot takes a step in the tangent direction of a point-GVG edge. Since by definition of the $R$-edge, the rod already lies in the tangent space of the point-GVG edge that defines the $R$-edge, the rod simply takes a step in the direction of the tangent space with fixed orientation.

As a result of the prediction step, the rod may break contact with the point-GVG edge that defines the $R$ edge being traced. In this case, let $r$ be the point on the rod that was in contact with the point-GVG edge prior to the prediction step. In this situation, the correction procedure has three steps: (1) using the point point-GVG corrector method with $r$ as a point robot, correct the point robot $r$ back to the point-GVG edge; (2) rotate the rod so that it is in the tangent space of the point-GVG edge; and (3) slide the rod along the tangent space of the point-GVG edge so that the inequalities of Equation 5 are satisfied. See Figure 10.

Now, consider the other case of when the rod does 


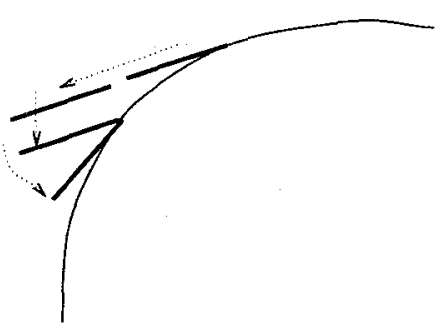

Fig. 10. Thick solid lines represent the prediction and correction steps of a rod as it traces out an $R$-edge defined by the pointGVG edge represented by the thin curved line.

not break contact with the point-GVG edge during the prediction phase. Let $r_{\text {before }}$ be the point on the rod where the rod intersected the point-GVG prior to the prediction step, let $r$ be the point on the rod that is in contact with the point-GVG edge after to the prediction step. If $r$ satisfies the inequalities of Equation 5, then the rod is already on an $R$-edge and thus there is no need to invoke a correction procedure. If $r$ does not satisfy these inequalities, treat $r_{\text {before }}$ as a point robot, and invoke the correction procedure outlined in the previous paragraph.

\subsubsection{Initiating and Terminating Conditions}

So far, it has been shown how to get on the rodHGVG and how to trace its components. The following two lemmas, whose proofs are omitted due to space limitations, list the conditions of when to terminate the tracing procedure.

LEMMA 6.2 The terminating conditions of a rod-GVG edge are either in the boundary of the environment or when the rod is equidistant to four obstacles, i.e., a rodGVG vertex.

Lemma 6.3 The terminating conditions of an $R$-edge are either on the boundary of the environment or when the rod is equidistant to three obstacles, i.e., a point on a rod-GVG edge.

Incremental construction of the rod-HGVG is akin to a graph search where the rod-GVG edges and the $R$-edges are the "edges" and the above described terminating conditions are the "nodes." The robot terminates exploration of the rod-HGVG when there are no more unexplored directions associated with any nodes. If the robot is looking for a particular destination whose coordinates are known, then the robot can invoke graph searching techniques, such as the A-star algorithm, to control the tracing procedure.

\subsection{Departability}

This section quickly reviews how the rod-HGVG is incrementally constructed. Incremental departability is a consequence of incremental departability of the pointGVG in the plane. The remaining point deals with com-

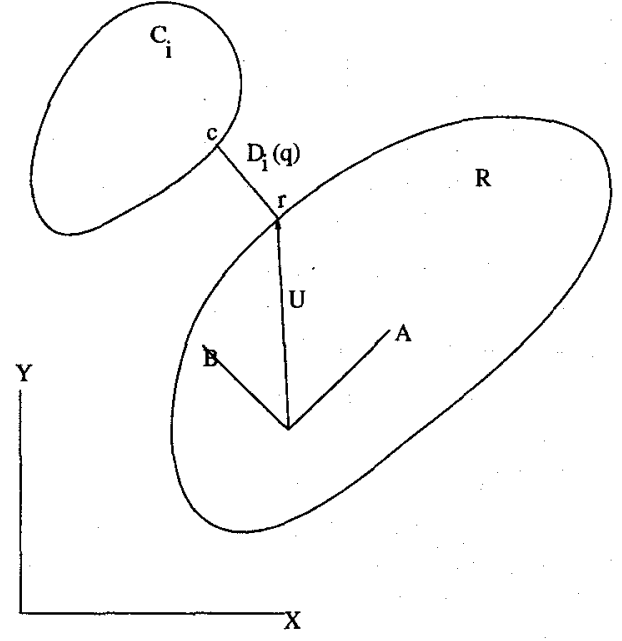

Fig. 11. Description of the variables

puting the gradient of $D$.

\section{Distance Gradient}

In actuality, the rod-distance function definition also applies to measuring distance between two convex sets. Therefore, this section is devoted to the gradient of the distance between two convex sets. The distance between the robot and a convex obstacle is simply the distance between the pair of closest points on the robot and obstacle. That is,

$$
D_{i}(q)=\min _{r \in R(q), c \in C_{i}}\|c-r\|
$$

measures the distance between a convex robot and a convex obstacle, where $q \in S E(2)$ and $R(q)$ are the set of points in $\mathbb{R}^{2}$ that the robot occupies. Note that this definition is identical to Equation (2).

Assume a world coordinate frame whose axes are $X$ and $Y$ and a body fixed coordinate frame on $R$ whose axes are $A$ and $B$. Let $(x, y)^{T}$ be the origin of the body fixed coordinates in the world coordinate frame and let $\theta$ denote the orientation of the body fixed coordinate frame with respect to the world coordinate frame. Let $c$ be the closest point on the obstacle $C_{i}$ to the robot $R$ and let $r$ be the closest point on the robot $R$ to the obstacle $C_{i}$. Finally, let $(a, b)^{T}$ be $r$ in the body fixed coordinate frame. See Figure 11. Therefore, the world coordinates of $r$ is

$$
\begin{aligned}
r & =\left[\begin{array}{l}
x \\
y
\end{array}\right]+\left[\begin{array}{rr}
\cos \theta & -\sin \theta \\
\sin \theta & \cos \theta
\end{array}\right]\left[\begin{array}{l}
a \\
b
\end{array}\right] \\
& =\left[\begin{array}{l}
x+a \cos \theta-b \sin \theta \\
y+a \sin \theta+b \cos \theta
\end{array}\right] .
\end{aligned}
$$

The distance $D_{i}(q)$ is $\left(\left(c^{x}-r^{x}\right)^{2}+\left(c^{y}-r^{y}\right)^{2}\right)^{\frac{1}{2}}$. 
First, consider the partial derivative with respect to $x$.

$$
\begin{aligned}
\frac{\partial D_{i}(q)}{\partial x}=\frac{1}{D_{i}(q)} & \left(\left(c^{x}-r^{x}\right)\left(\frac{\partial c^{x}}{\partial x}-\frac{\partial r^{x}}{\partial x}\right)\right. \\
& \left.+\left(c^{y}-r^{y}\right)\left(\frac{\partial c^{y}}{\partial x}-\frac{\partial r^{y}}{\partial x}\right)\right)
\end{aligned}
$$

From Equation (9),

$$
\begin{aligned}
& \frac{\partial r^{x}}{\partial x}=1+\frac{\partial a}{\partial x} \cos \theta-\frac{\partial b}{\partial x} \sin \theta \\
& \frac{\partial r^{y}}{\partial x}=\frac{\partial a}{\partial x} \sin \theta+\frac{\partial b}{\partial x} \cos \theta
\end{aligned}
$$

Substitute the above into $\frac{\partial D_{i}(q)}{\partial x}$ in Equation 10 .

$$
\begin{aligned}
\frac{\partial D_{i}(q)}{\partial x}= & \frac{1}{D_{i}(q)}\left(\left(c^{x}-r^{x}\right)\left(\frac{\partial c^{x}}{\partial x}-1-\frac{\partial a}{\partial x} \cos \theta+\frac{\partial b}{\partial x} \sin \theta\right)\right. \\
& \left.\quad+\left(c^{y}-r^{y}\right)\left(\frac{\partial c^{y}}{\partial x}-\frac{\partial a}{\partial x} \sin \theta-\frac{\partial b}{\partial x} \cos \theta\right)\right) \\
= & \frac{1}{D_{i}(q)}\left\langle\left[\begin{array}{l}
\left(c^{x}-r^{x}\right) \\
\left(c^{y}-r^{y}\right)
\end{array}\right]\right. \\
& \left.\left(\left[\begin{array}{c}
-1 \\
0
\end{array}\right]+\left[\begin{array}{l}
\frac{\partial c^{x}}{\partial x} \\
\frac{\partial c^{y}}{\partial x}
\end{array}\right]-\left[\begin{array}{l}
\frac{\partial a}{\partial x} \cos \theta-\frac{\partial b}{\partial x} \sin \theta \\
\frac{\partial a}{\partial x} \sin \theta+\frac{\partial b}{\partial x} \cos \theta
\end{array}\right]\right)\right\rangle
\end{aligned}
$$

Note that the vector $c-r$ is orthogonal to the tangent space of the boundary of the obstacle at $c$, as well as to the tangent space of the boundary of the robot at $r$. Note that $\left[\frac{\partial c^{2}}{\partial x}, \frac{\partial c^{y}}{\partial x}\right]^{T}$ is an element in the tangent space of the boundary of the obstacle and that $\left[\frac{\partial a}{\partial x} \cos \theta-\right.$ $\left.\frac{\partial b}{\partial x} \sin \theta, \frac{\partial a}{\partial x} \sin \theta+\frac{\partial b}{\partial x} \cos \theta\right]^{T}$ is an element in the tangent space of the boundary of the robot. Therefore, the dot products of $c-r$ with both of these vectors is zero and thus we have:

$$
\frac{\partial D_{i}(q)}{\partial x}=\frac{1}{D_{i}(q)}\left(r^{x}-c^{x}\right)
$$

Using similar analysis, we can easily conclude that

$$
\frac{\partial D_{i}(q)}{\partial y}=\frac{1}{D_{i}(q)}\left(r^{y}-c^{y}\right)
$$

Finally, consider $\frac{\partial D_{i}}{\partial \theta}$.

$$
\begin{aligned}
\frac{\partial D_{i}(q)}{\partial \theta}=\frac{1}{D_{i}(q)} & \left(\left(c^{x}-r^{x}\right)\left(\frac{\partial c^{x}}{\partial \theta}-\frac{\partial r^{x}}{\partial \theta}\right)\right. \\
& \left.+\left(c^{y}-r^{y}\right)\left(\frac{\partial c^{y}}{\partial \theta}-\frac{\partial r^{y}}{\partial \theta}\right)\right)
\end{aligned}
$$

From Equation (9),

$$
\begin{aligned}
& \frac{\partial r^{x}}{\partial \theta}=\frac{\partial a}{\partial \theta} \cos \theta-a \sin \theta-\frac{\partial b}{\partial \theta} \sin \theta-b \cos \theta \\
& \frac{\partial r^{\nu}}{\partial \theta}=\frac{\partial a}{\partial \theta} \sin \theta+a \cos \theta+\frac{\partial b}{\partial \theta} \cos \theta-b \sin \theta
\end{aligned}
$$

Substitute the above into $\frac{\partial D_{i}(g)}{\partial \theta}$ in Equation (14).

$$
\begin{aligned}
& \frac{\partial D_{i}}{\partial \theta}=\frac{1}{D_{i}(q)}\left(( c ^ { x } - r ^ { x } ) \left(\frac{\partial c^{x}}{\partial \theta}-\frac{\partial a}{\partial \theta} \cos \theta\right.\right. \\
& \left.+a \sin \theta+\frac{\partial b}{\partial \theta} \sin \theta+b \cos \theta\right) \\
& +\left(c^{y}-r^{y}\right)\left(\frac{\partial c^{y}}{\partial \theta}-\frac{\partial a}{\partial \theta} \sin \theta\right. \\
& \left.\left.-a \cos \theta-\frac{\partial b}{\partial \theta} \cos \theta+b \sin \theta\right)\right) \\
& =\frac{1}{D_{i}(q)}\left\langle\left[\begin{array}{l}
\left(c^{x}-r^{x}\right) \\
\left(c^{y}-r^{y}\right)
\end{array}\right],\left(\left[\begin{array}{l}
a \sin \theta+b \cos \theta \\
b \sin \theta-a \cos \theta
\end{array}\right]\right.\right. \\
& \left.\left.+\left[\begin{array}{l}
\frac{\partial c^{*}}{\partial \theta} \\
\frac{\partial c^{y}}{\partial \theta}
\end{array}\right]-\left[\begin{array}{l}
\frac{\partial a}{\partial \theta} \cos \theta-\frac{\partial b}{\partial \theta} \sin \theta \\
\frac{\partial a}{\partial \theta} \sin \theta+\frac{\partial b}{\partial \theta} \cos \theta
\end{array}\right]\right)\right\rangle \\
& =\frac{1}{D_{i}(q)}\left\langle\left[\begin{array}{l}
\left(c^{x}-r^{x}\right) \\
\left(c^{y}-r^{y}\right)
\end{array}\right],\left[\begin{array}{l}
a \sin \theta+b \cos \theta \\
b \sin \theta-a \cos \theta
\end{array}\right]\right\rangle \\
& =\frac{1}{D_{i}(q)}\left[\begin{array}{l}
c^{x}-r^{x} \\
c^{y}-r^{y}
\end{array}\right]^{T}\left[\begin{array}{cc}
0 & 1 \\
-1 & 0
\end{array}\right]\left[\begin{array}{cc}
\cos \theta & -\sin \theta \\
\sin \theta & \cos \theta
\end{array}\right]\left[\begin{array}{l}
a \\
b
\end{array}\right] \\
& =\frac{1}{D_{i}(q)}\left[\begin{array}{l}
c^{x}-r^{x} \\
c^{y}-r^{y}
\end{array}\right]^{T}\left[\begin{array}{cc}
0 & 1 \\
-1 & 0
\end{array}\right]\left[\begin{array}{l}
U^{x} \\
U^{y}
\end{array}\right] \\
& =\frac{1}{D_{i}(q)}(c-r) \times U \\
& =U \times \nabla d_{i}(r),
\end{aligned}
$$

where $U$ is described in world coordinates. That is, $U=\operatorname{Rot}(\theta)[a, b]^{T}$. Therefore, the gradient is

$$
\left[\begin{array}{c}
\nabla d_{i}(r) \\
U \times \nabla d_{i}(r)
\end{array}\right]
$$

where $\nabla d_{i}(r)$ is a $3 \times 1$ vector that is the gradient of a single object distance function evaluated at $r$ and $U$ is as described above. Current work includes generalizing this result to $S E(3)$.

It is interesting to note that this gradient definition reflects the lack of bi-invariance of all metrics in $S E(2)$ and $S E(3)$ [13]. A left-invariant metric in $S E(3)$ is one for which given any two points $p_{1}, p_{2} \in S E(3)$, the distance between these points, $d\left(p_{1}, p_{2}\right)$, is the same as $d\left(T p_{1}, T p_{2}\right)$ for all $T \in S E(3)$. This means, changing the location of the world coordinate frame does not change the distance between two points in $S E(3)$. A right-invariant metric in $S E(3)$ is one for which given any two two points $p_{1}, p_{2} \in S E(3)$, the distance between these points, $d\left(p_{1}, p_{2}\right)$, is the same as $d\left(p_{1} T, p_{2} T\right)$ for all $T \in S E(3)$. This means that changing the location of the body fixed coordinate frame does not affect the distance between two points in $S E(3)$. It was shown in [13] that no metric in $S E(3)$ can be both left-invariant and right-variant, i.e., no metric in $S E(3)$ can be bi-invariant. Note that the gradient in Equation (16) depends upon the choice of a body-fixed coordinate frame; this reflects the lack of bi-invariance in $S E(2)$ and $S E(3)$. 
Recall the example in Section 6.1 of the rod accessing the rod-HGVG in Figure 8. The choice of base frame on the robot affects the path traced out by the gradient, but it does not affect the location of the local maxima.

Also note that the definition of the rod-HGVG uses the gradient in Equation (16). This means that the rodHGVG is dependent upon the choice of the body-fixed frame. In [4], the rod-HGVG was defined in terms of a translational gradient, $\tilde{\nabla} D_{i}(q)$, which is a $3 \times 1$ unit vector $\left[\nabla d_{i}(r) 0\right]^{T}$, where $r$ is the closest point on the $\operatorname{rod} R$ to obstacle $C_{i}$.

\section{Conclusion}

This paper introduces the incremental construction procedure for a roadmap called the rod hierarchical generalized Voronoi graph. The rod-HGVG is defined in terms of line of sight workspace distance information, which is the upper bound to what sensors can readily provide. This makes the rod-HGVG well suited for sensor based motion planning. Although this incremental construction procedure was specifically developed for sensor based implementation of a rod, it can be used for classical motion planning as well. Simulations of this method are underway.

The ultimate goal of this research program is to enable highly articulated robots equipped with sensors to explore unknown environments, via construction of a roadmap. The first step towards this goal was the development of the point hierarchical generalized Voronoi graph (point-HGVG) and its incremental construction procedure. The point-HGVG is a roadmap for robots that can be modeled as a point; the point-HGVG results are general to three-dimensional workspaces. Based on the point-HGVG results, the next step was the development of the rod-HGVG and the introduction of its incremental construction procedure, described in this paper. The next step is to extend the results of the rod roadmap to that of a convex set, which in turn will be extended to the development of a roadmap for a chain of convex sets which model a highly articulated robot.

A key feature of the rod-HGVG (and future roadmap definitions) is that it is defined in terms of work space distance measurements. Nevertheless the rod-HGVG is a subset of $S E(2)$, and thus the gradient (really, the differential) of the distance function $D_{i}(q)$ requires addition analysis because $D_{i}(q)$ is a non-Euclidean metric. A key result of this paper is the derivation of the gradient (differential) of $D_{i}(q)$. (Note that this gradient reflects the lack of bi-invariance property of distance metrics in $S E(2)$ and $S E(3)$.)

The above described gradient technique is useful in path planning for non-holonomic robots, such as a mobile robot with a minimum turning radius. Replac- ing the standard Euclidean metric with one that measures distances only along feasible paths of the robot gives a more realistic measure of proximity to obstacles, accounting for the constraints on the robot's motion. Planning using a GVG constructed with this feasible path metric results in lower complexity paths for the robot [9]. Details on the differentiability of a feasible path metric for car-like robots are discussed in [10].

\section{References}

[1] J.F. Canny and M.C. Lin. An Opportunistic Global Path Planner. Algorithmica, 10:102-120, 1993.

[2] H. Choset and J.W. Burdick. Sensor Based Planning, Part I The Generalized Voronoi Graph. In Proc. IEEE Int. Conf. an Robotics and Automation, Nagoya, Japan, 1995.

[3] H. Choset and J.W. Burdick. Sensor Based Planning, Part II: Incremental Construction of the Generalized Voronoi Graph. In Proc. IEEE Int. Conf. on Robotics and Automation, Nagoya, Japan, 1995.

[4] H. Choset and J.W. Burdick. Sensor Based Planning for a Planar Rod Robot. In Proc. IEEE Int. Conf. on Robotics and Automation, Minneapolis, MN, 1996.

[5] H. Choset and J.W. Burdick. Sensor Based Planning: The Hierarhical Generalized Voronoi Graph. In Proc. Workshop on Algorithmic Foundations of Robotics, Toulouse, France, 1996.

[6] J. Cox and C.K. Yap. On-line Motion Planning: Case of a Planar Rod. Annals of Mathematics and Artifical Intelligence, 3:1-20, 1991.

[7] H.B. Keller. Lectures on Numerical Methods in Bifurcation Problems. Tata Institute of Fundamental Research, Bombay, India, 1987.

[8] V. Lumelsky and A. Stepanov. Path Planning Strategies for Point Mobile Automaton Moving Amidst Unknown Obstacles of Arbitrary Shape. Algorithmica, 2:403-430, 1987.

[9] Brian Mirtich and John Canny. A skeleton-based planner for the two-axle car. IEEE Transactions on Robotics and Automation, 1997.

[10] Philippe Moutarlier, Brian Mirtich, and John Canny. Shortest paths for a car-like robot to manifolds in configuration space. International Journal of Robotics Research, 15(1):3660, February 1996.

[11] C. Ó'Dúnlaing, M. Sharir, and C.K. Yap. Generalized Voronoi Diagrams for Moving a Ladder. I: Topological Analysis. Communications on Pure and Applied Mathematics, 39:423-483, 1986.

[12] C. Ó'Dúnlaing and C.K. Yap. A "Retraction" Method for Planning the Motion of a Disc. Algorithmica, 6:104-111, 1985.

[13] F. C. Park. Distance Metrics on the Rigid-body Motions with Applications to Mechanism Desgin. ASME Journal of Mechanical Design, 1995.

[14] N.S.V. Rao, S. Kareti, W. Shi, and S.S. Iyenagar. Robot Navigation in Unknown Terrains: Introductory Survey of Non-Heuristic Algorithms. Oak Ridge National Laboratory Technical Report, ORNL/TM-12410:1-58, July 1993.

[15] E. Rimon and J.F. Canny. Construction of C-space Roadmaps Using Local Sensory Data - What Should the Sensors Look For? In Proc. IEEE Int. Conf. on Robotics and Automation, pages 117-124, San Diego, CA, 1994.

[16] J.A. Thorpe. Elementary Topics in Differential Geometry. Springer-Verlag, 1985. 